\title{
DESARROLLO Y PREDICCIÓN DE SINTÉTICOS DE CRUZAS DOBLES DE MAÍZ1
}

\author{
Jaime Sahagún Castellanos ${ }^{2}$, J. Enrique Rodríguez Pérez ${ }^{2}$, Aureliano Peña Lomeli ${ }^{2}$
}

\begin{abstract}
RESUMEN
Desarrollo y predicción de sintéticos de cruzas dobles de maíz. Las variedades híbridas $(\mathrm{VH})$ han sido clasificadas entre las que, en condiciones óptimas, mejor explotan el potencial genético de varias especies agrícolas. Sin embargo, la siembra de su $\mathrm{F}_{2}$, o de una generación posterior, no regenera la VH sino una población similar a una variedad sintética (VS), particularmente si la VH es una cruza doble (CD). Usualmente, una VS se caracteriza por una plasticidad poblacional que le permite ser una buena alternativa para la agricultura de escasos insumos. Considerando que la formación de una VS con cruzas dobles puede ser una vía para estudiar la capacidad de un mayor número de líneas para formar VS superiores y que es importante contribuir al acervo teórico de la predicción de las medias genotípicas de las VS, sus fórmulas de predicción (FP) de VS, fueron desarrolladas con $n$ cruzas dobles. Tres fórmulas (FPAA) se basaron en la evaluación de las cruzas directas entre progenitores [las $n$ cruzas dobles (CD), sus $2 \mathrm{n}$ cruzas simples (CS), o sus $4 \mathrm{n}$ líneas] y las poblaciones que resultan del apareamiento aleatorio (PAA) en aislamiento de los individuos de cada progenitor. En las otras tres fórmulas (FPR), de cada PAA se evaluaron dos partes: La producida por autofecundación y la restante. Los seis predictores fueron insesgados, pero entre métodos y entre progenitores, los FPR y las líneas tuvieron, respectivamente, la menor varianza. La eficiencia relativa de las FPR con relación a las FPAA tuvo una superioridad que varió desde 8 a $40 \%$ para diferentes combinaciones de números de progenitores y plantas por progenitor. Formalmente la estimación que se pudo hacer con el menor número de unidades experimentales se basa en $\mathrm{CD}$, aunque su varianza, fue la más alta para $\mathrm{n} \geq 2$. Sin embargo, para $\mathrm{n}=1$ y un número constante de unidades experimentales el estimador basado en CD fue el más preciso.
\end{abstract}

Palabras claves: predicción de sintéticos, precisión, híbridos, arreglo genotípico.

\begin{abstract}
Development and prediction of double cross synthetics of mayze. Hybrid varieties (VH) have been identified among the best, under optimal conditions, to exploit the genetic potential of several crop species. Sowing $\mathrm{F}_{2}$ plantings or more advanced seed, does not regenerate the $\mathrm{VH}$. A population similar to a synthetic variety (VS) is obtained instead, particularly if the hybrid is a double cross. A VS is characterized by possessing a population buffering which makes, it to be adequate for low input agricultural conditions. Considering that building up VS with double crosses is a procedure assessing the capacity of a higher number of lines to form superior VS, and since it is important to increase the theoretical knowledge about performance prediction, six prediction formulae (FP) for a VS built-up with $n$ double were derived. Three formulas (FPAA) were based on the field evaluation of the progenies produced by the direct crosses among parents [the $n$ double-cross hybrids (CD), the involved $2 n$ single crosses (CS), or the $4 n$ lines] and the populations produced by randomly mating (PAA) in isolation. The individuals representing each parent. In the additional three FPR, each PAA population was evaluated in two parts: one produced by selfing and the remaining. All six were unbiased, but between types and among parents the FPR methods and lines, respectively, showed the lowest variances. The superiority of the FPR in relation with the FPAA methods ranked from 8 to $40 \%$. In addition, for $n \geq 2$, the estimation that required less plots was based on $\mathrm{CD}$, but the precision was the lowest. On the other hand, for $n=1$ and a fixed number of plots the most precise estimator was based on CD as well.
\end{abstract}

Key words: synthetic prediction, precision, hybrids, genotypic array.

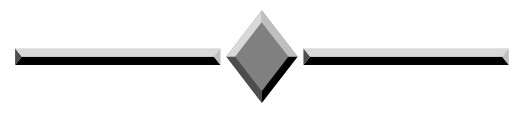

1 Recibido: 19 de abril, 2004. Aceptado: 7 de febrero, 2005. Presentado en la XLIX Reunión Anual del PCCMCA. La Ceiba, Honduras, 2003.

2 Programa Universitario de Investigación y Servicio en Olericultura. Depto. de Fitotecnia de la Universidad Autónoma Chapingo. Km 38.5 Carr. México-Texcoco; 56230. Chapingo, Méx. E-mail: jsahagun@taurus1.chapingo.mx. 


\section{INTRODUCCIÓN}

A diferencia de los híbridos (cruzas simples, cruzas triples y cruzas dobles), una variedad sintética (VS) genera su semilla por el apareamiento aleatorio, en aislamiento, de los individuos que la constituyen. Esta característica de los sintéticos tiene consecuencias en su estructura genotípica, que a su vez determina rasgos de importancia en su comportamiento. Por ser una población altamente heterocigótica y heterogénea, una VS debe poseer una considerable plasticidad poblacional (Allard y Bradshaw 1964). Esta característica de la VS le permite afrontar, muchas veces exitosamente, los cambios del ambiente debidos a diferencias en lugar, en el tiempo meteorológico (cantidad y distribución de la precipitación, temperatura, humedad), en incidencia de plagas y enfermedades, y en el manejo agronómico (en niveles de aplicación de fertilizantes, herbicidas, riego). Esta característica de una VS ha hecho que se le considere adecuada para la agricultura de bajos insumos.

Por otra parte, el apareamiento aleatorio en aislamiento de los individuos de una VS debe producir una arreglo genotípico que alcanza la estabilidad o equilibrio en sólo una generación; y esto significa que una VS al reproducirse produce la semilla que la regenerará. En los híbridos esto no sucede así.

En una parcela de producción sembrada con una variedad híbrida, la semilla que se produce genera una población que, en relación al híbrido, experimenta una reducción en el rendimiento por unidad de superficie (González et al. 1990, Villanueva et al. 1994). En realidad, la población que genera el apareamiento aleatorio de la $\mathrm{F}_{2}$, o de una generación más avanzada, de un híbrido, debe tener el arreglo genotípico de una variedad sintética. Así, si bien con este procedimiento se elimina el problema de adquisición de la semilla híbrida $\mathrm{F}_{1}$, se experimentará una reducción en la media genotípica.

El desarrollo de una VS requiere la identificación de un grupo de progenitores que tengan, además de otros atributos, un buen balance de aptitudes combinatoria general y específica. Similarmente, las líneas con que se construyen los diferentes tipos de híbridos debieron haber mostrado entre otras cualidades, un buen nivel de estas aptitudes. Esto significa que tales líneas deben tener buen potencial para ser progenitores de una VS.

Uno de los problemas que tiene el desarrollo de VS es el de la predicción de cada una de las numerosas variedades que de este tipo pueden formarse con $\mathrm{n}$ progenitores potenciales. Si bien es posible reducir la magnitud de este problema mediante la evaluación de los n progenitores y de sus cruzas (Wright 1922, Wright
1981, Márquez-Sánchez 1992a, Sahagún-Castellanos 1998) a medida que $n$ aumenta, la probabilidad de encontrar una VS superior también debe incrementarse, aunque el número de materiales a ser evaluados $[\mathrm{n}(\mathrm{n}+1) / 2 ;$ los $\mathrm{n}$ progenitores y sus $\mathrm{n}(\mathrm{n}-1) / 2$ sus cruzas] también aumenta. Una forma de reducir este número puede basarse en el uso de progenitores que en lugar de líneas sean cruzas dobles; así, cada cruza doble debe representar cuatro progenitores iniciales [el número mínimo de progenitores de una VS según Busbice (1970)]. Sin embargo, la formación de VS por esta vía presenta ángulos inéditos que deben ser estudiados. Por ejemplo, las propiedades que tienen las líneas como progenitores no necesariamente las deben tener sus cruzas dobles; para $\mathrm{n}$ cruzas dobles formadas con $4 \mathrm{n}$ líneas cuyos coeficiente de endogamia y coancestría son iguales a uno y cero, respectivamente, el coeficiente de endogamia y el de las cruzas entre ellas deben ser iguales a cero.

Por las consideraciones anteriores y porque es importante contribuir al acervo teórico de la predicción de las VS, se planeó la presente investigación cuyo objetivo fue derivar y estudiar fórmulas para predecir la media genotípica de las VS cuyos progenitores son híbridos de cruza doble.

\section{MÉTODOS Y MARCO TEÓRICO}

Con el modelo de población que se asumió, se pretendió representar de la mejor manera la realidad de las VS de especies cultivadas como el maíz (Zea mays L.), la cebolla (Allium cepa), tomate de cáscara (Physalis ixocarpa Brot.) y otras especies diploides. En este sentido, la VS se estudió como si fuera una población de individuos diploides y bisexuales que se reproducen por apareamiento aleatorio, en ausencia de mutación, migración, selección, efectos maternos y deriva genética.

Respecto a la formación de las $n$ cruzas dobles se consideraron $4 n$ líneas denominadas $\mathrm{i}_{1} \mathrm{i}_{2}, \mathrm{i}_{3} \mathrm{i}_{4}, \mathrm{i}_{5} \mathrm{i}_{6}$ e $\mathrm{i}_{7} \mathrm{i}_{8}$ $(i=1,2, \ldots, n)$, y el genotipo de la planta $x(x=1,2,3, \ldots, y)$ de la línea $\mathrm{i}_{(2 \mathrm{k}-1)} \mathrm{i}_{(2 \mathrm{k})}(\mathrm{k}=1,2,3,4)$ se expresó como $\mathrm{A}_{\mathrm{xi}(2 \mathrm{k}-1)} \mathrm{A}_{\mathrm{xi}(2 \mathrm{k})}$ (el paréntesis del tercer subíndice sólo se usó cuando éste involucró más de un término). Con las $4 \mathrm{n}$ líneas se formaron $2 \mathrm{n}$ cruzas simples en las formas $i_{1} i_{2} \times i_{3} i_{4}$ e $i_{5} i_{6} \times i_{7} i_{8}$. Con estas cruzas simples se consideró la construcción de $n$ cruzas dobles en la forma $\left[\mathrm{i}_{1} \mathrm{i}_{2} \times \mathrm{i}_{3} \mathrm{i}_{4}\right] \times\left[\mathrm{i}_{5} \mathrm{i}_{6} \times \mathrm{i}_{7} \mathrm{i}_{8}\right]$. Para generar una cruza simple o doble se consideró que cada línea o cruza simple involucrada, respectivamente, participó con el mismo número de plantas.

En lo que sigue se usará los conceptos y notación (o sus extensiones) que se han utilizado en estudios 
previos (Busbice 1969, 1970, Márquez-Sánchez 1992b, Sahagún y Villanueva 1997). Así, se consideró que cada cruza doble fue representada por $m$ plantas; y el genotipo de la planta $\mathrm{p}(\mathrm{p}=1,2, \ldots, \mathrm{m})$ de la cruza doble $\mathrm{i}$ $(\mathrm{i}=1,2, \ldots, \mathrm{n})$ fue expresado como $\mathrm{A}_{\text {pik }} \mathrm{A}_{\text {piv }} \mathrm{v}(\mathrm{k}=1,2$, $3,4 ; \mathrm{v}=5,6,7,8)$. Por esta razón, el arreglo genotípico de la población formada por los $\mathrm{m}$ individuos de cada cruza doble, denominada sintético cero, se expresó como

$$
\mathrm{AGS}-0=\frac{1}{16 \mathrm{mn}} \sum_{\mathrm{p}} \sum_{\mathrm{i}} \sum_{\mathrm{k}} \sum_{\mathrm{v}} \mathrm{A}_{\mathrm{pik}} \mathrm{A}_{\mathrm{piv}}
$$

El arreglo genotípico del sintético uno, la población que produce el cruzamiento de cada progenitor con todas las demás, fue

$$
\text { AGS }-1=\frac{1}{(8 m)^{2} n(n-1)} \sum_{\text {p }} \sum \sum \sum \sum \sum \sum \mathrm{A}_{\mathrm{i} \text { ir }} \mathrm{A}_{\mathrm{qj}}
$$

En la Ecuación 2, y en lo subsiguiente, $\mathrm{r}, \mathrm{s}=1,2$, $3, \ldots ., 8$. Respecto a la generación siguiente, el arreglo genotípico de la población que produce el apareamiento aleatorio de los individuos del sintético uno, llamado sintético dos (Busbice 1970), que es el que corresponde al de la VS, tiene que ser de la forma

$$
\text { AGS }-2=\frac{1}{(8 m n)^{2}} \sum_{\mathrm{p}} \sum_{\mathrm{q}} \sum_{\mathrm{i}} \sum_{\mathrm{j}} \sum_{\mathrm{r}} \sum_{\mathrm{s}} \mathrm{A}_{\mathrm{pir}} \mathrm{A}_{\mathrm{qjs}}
$$

Si el valor genotípico de se representa por en la Ecuación 3, la media genotípica de los sintéticos cero, uno y dos (Ecuaciones 1, 2, 3) son, respectivamente:

$$
\begin{aligned}
& \mathrm{Y}_{0 \mathrm{CD}}=\frac{1}{16 \mathrm{mn}} \sum_{\mathrm{p} \text { i k v }} \sum_{\mathrm{v}} \sum_{\text {pir, pis }} \\
& \mathrm{Y}_{1 \mathrm{CD}}=\frac{1}{(8 \mathrm{~m})^{2} \mathrm{n}(\mathrm{n}-1)} \sum_{\mathrm{p}} \sum_{\mathrm{q}} \sum_{\mathrm{i} \neq \mathrm{j} \text { r s }} \sum_{\mathrm{s}} \mathrm{Y}_{\text {pir, qjs }} \\
& \mathrm{Y}_{2 \mathrm{CD}}=\frac{1}{(8 \mathrm{mn})^{2}} \sum_{\mathrm{p} \mathrm{q}} \sum_{\mathrm{i}} \sum_{\mathrm{i}} \sum_{\mathrm{r} \text { r s }} \mathrm{Y}_{\text {pir, qjs }}
\end{aligned}
$$

Como en estudios previos (Wright 1981, Sahagún y Villanueva 1997, Sahagún-Castellanos 1998), la predicción de la media genotípica de una VS se hizo con base en descomposiciones del total de los valores genotípicos de los individuos que forman la VS (Ecuación 6). La idea central es que cada descomposición se haga en partes que puedan ser identificadas, formadas y evaluadas en experimentos de campo para generar los estimadores correspondientes.

Para caracterizar desde un punto de vista estadístico a cada estimador de la media genotípica de una VS se determinó su sesgo, su varianza (Wricke y Weber 1986, Sahagún-Castellanos 1998). Adicionalmente se calculó la eficiencia relativa entre cada dos tipos de estimadores en la base de un mismo número de unidades experimentales; esta eficiencia se calculó como el cociente de la varianza de un estimador entre la del otro.

\section{RESULTADOS Y DISCUSIÓN}

El total de los valores genotípicos de la VS (Ecuación 6) se puede descomponer en dos partes, una de ellas involucra el lado derecho de la Ecuación 5. Esto es:

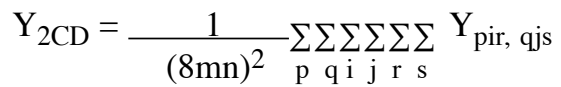

$$
\begin{aligned}
& =\frac{1}{(8 m n)^{2}}\left[\sum_{\mathrm{p}} \sum_{\mathrm{q} i \neq \mathrm{j}} \sum_{\mathrm{r} \text { s }} \sum_{\mathrm{p}} \mathrm{Y}_{\mathrm{pir}, \mathrm{qjs}}+\sum_{\mathrm{pq}} \sum_{\mathrm{i}} \sum_{\mathrm{r} s \mathrm{~s}} \mathrm{Y}_{\mathrm{pir}, \mathrm{qis}}\right]^{(7)}
\end{aligned}
$$

El segundo término del lado derecho de la Ecuación 7 es el total de los valores genotípicos de los individuos de las poblaciones formadas por el apareamiento aleatorio en aislamiento de los individuos de cada cruza doble. Si la media genotípica de estas poblaciones se simboliza por y se considera la Ecuación 5, la Ecuación 7 se puede escribir también como:

$$
\begin{aligned}
\mathrm{Y}_{2 \mathrm{CD}} & =\frac{1}{(8 \mathrm{mn})^{2}}\left[(8 \mathrm{~m})^{2} \mathrm{n}(\mathrm{n}-1) \mathrm{Y}_{1 \mathrm{CD}}+(8 \mathrm{~m})^{2} \mathrm{n} \mathrm{R}_{\mathrm{CD}}\right] \\
& =\frac{\mathrm{n}-1}{\mathrm{n}} \mathrm{Y}_{1 \mathrm{CD}}+\frac{1}{\mathrm{n}} \mathrm{R}_{\mathrm{CD}} \\
& =\mathrm{Y}_{1 \mathrm{CD}}-\left(\mathrm{Y}_{1 \mathrm{CD}}-\mathrm{R}_{\mathrm{CD}}\right) / \mathrm{n}
\end{aligned}
$$

De acuerdo con la Ecuación 8, la predicción de la VS formada con $\mathrm{n}$ cruzas dobles se hace con base en la estimación de la media de las progenies que genera los cruzamientos entre todas las cruzas dobles $\left(\mathrm{Y}_{1 \mathrm{CD}}\right)$ y de la media de las poblaciones que genera el apareamiento aleatorio en aislamiento de los individuos de cada una de estas cruzas dobles $\left(\mathrm{R}_{\mathrm{CD}}\right)$. Este resultado no es sorpresivo, es consistente con las expresiones teóricas más generales, derivadas por diferentes vías por Wright (1981) y Sahagún-Castellanos (1998).

Otra descomposición del total de los valores genotípicos presentes en $\mathrm{Y}_{2 \mathrm{CD}}$ se basa en la identificación de tres partes en el segundo término de la Ecuación 7, que corresponden a los individuos que produce: 1) La autofecundación de los individuos que representan las líneas que forman cada cruza doble; 2) El cruzamiento entre individuos de una misma línea, y 3) El cruzamiento entre individuos de líneas diferentes. Así, con base en algunos de los subíndices siguientes: 

$\mathrm{a}, \mathrm{b}=1,2$
$\mathrm{g}, \mathrm{h}=7,8$
$\mathrm{e}^{\mathrm{c}}=1,2,3,4,7,8$
c, $d=3,4$
$\mathrm{a}^{\mathrm{c}}=3,4,5,6,7,8$
$\mathrm{g}^{\mathrm{c}}=1,2,3,4,5,6$
e, $f=5,6$
$c^{c}=1,2,5,6,7,8$
$\mathrm{u}, \mathrm{v}=5,6,7,8$

la descomposición referida es expresable como

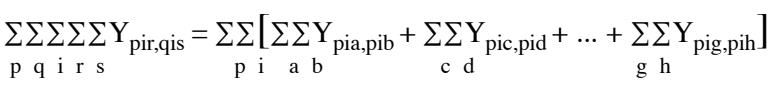

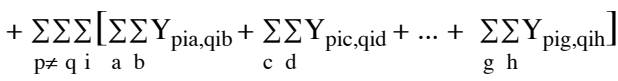

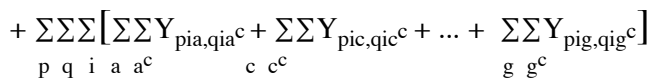

Los valores genotípicos de la segunda y tercera partes de esta expresión sólo corresponden a cruzamientos entre individuos de una misma cruza doble. Así, si $\mathrm{S}_{\mathrm{L}}$ y $\mathrm{R}_{\mathrm{CD}}$ representan la media genotípica de las progenies que produce la autofecundación de las líneas y el cruzamiento entre los individuos de cada cruza doble,

$$
\underset{\text { p q i r s }}{\sum \sum \sum \sum \sum \mathrm{Y}_{\text {pir,qis }}=16 \mathrm{mn}_{\mathrm{L}}+\left[16 \mathrm{~m}(\mathrm{~m}-1) \mathrm{n}+48 \mathrm{~m}^{2} \mathrm{n}\right] \mathrm{R}_{\mathrm{CD}}}
$$

Por lo tanto, con la substitución de este resultado en la Ecuación 7 y la consideración de la Ecuación 5, la media genotípica de la VS también puede expresarse como

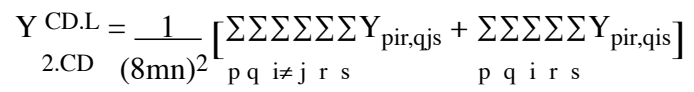

$$
\begin{aligned}
& \left.=11(8 m)^{2} n(n-1) Y_{1 C D}+16 m n S_{L}+\left[16 m(m-1) n+48 m^{2} n\right] R^{\prime}{ }_{C D}\right\} \\
& (8 \mathrm{mn})^{2} \\
& =\mathrm{Y}_{1 \mathrm{CD}}-\left(\mathrm{Y}_{1 \mathrm{CD}}-\mathrm{R}^{\prime}{ }_{\mathrm{CD}}\right) / \mathrm{n}-\left(\mathrm{R}^{\prime}{ }_{\mathrm{CD}}-\mathrm{S}_{\mathrm{L}}\right) / 4 \mathrm{mn}
\end{aligned}
$$

Como cada cruza doble se construye con dos cruzas simples, se investigó la posibilidad de expresar la media de la VS en términos de las 2 n cruzas simples. Respecto a las cuatro cruzas simples involucradas en cada dos cruzas dobles diferentes, resulta que:

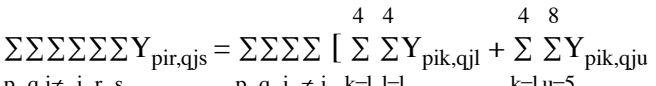

$$
\begin{aligned}
& \text { p q i } \neq \mathrm{jrs}_{\mathrm{r}} \quad \text { p q i } \neq \text { j } \quad \mathrm{k}=1 \mathrm{l}=1 \quad \mathrm{k}=\mathrm{l} u=5 \\
& \left.+\sum_{\mathrm{u}=5}^{8} \sum_{\mathrm{l}=\mathrm{l}}^{4} \mathrm{Y}_{\text {piu,qjl }}+\stackrel{8}{\sum} \stackrel{8}{\sum} \mathrm{Y}_{\text {piu,qju }}\right]
\end{aligned}
$$

Además, para las dos cruzas simples de una misma cruza doble,

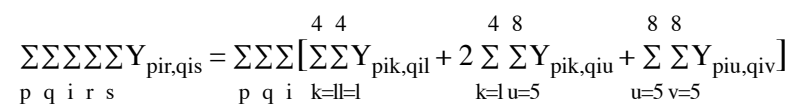

Para estas dos descomposiciones, sean $\mathrm{Y}_{1 \mathrm{CS}}$ y $\mathrm{R}_{\mathrm{CS}}$ la media genotípica de las progenies que producen todas las cruzas entre cruzas simples y la media genotípica de las $2 \mathrm{n}$ poblaciones que forma el apareamiento aleatorio en aislamiento de los individuos de cada cruza simple, respectivamente. De acuerdo con las consideraciones anteriores y con la Ecuación 7, la media genotípica de la VS también es expresable como

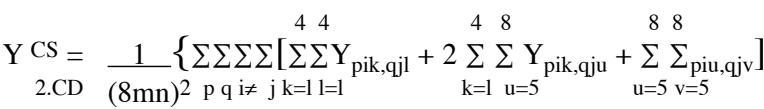

$$
\begin{aligned}
& \begin{array}{llrrr}
4 & 4 & 4 & 8 & 8
\end{array} \\
& \left.+\sum \Sigma \sum\left[\sum \sum \mathrm{Y}_{\text {pik,qil }}+2 \Sigma \sum \mathrm{Y}_{\text {pik,qiu }}+\sum \sum \mathrm{Y}_{\text {piu,qiv }}\right]\right\} \\
& \text { p q i } k=1 \mathrm{l}=1 \quad \mathrm{k}=1 \mathrm{u}=5 \quad \mathrm{u}=5 \mathrm{v}=5 \\
& =\frac{1}{(8 m n)^{2}}\left\{\left[m^{2} n(n-1)(64)+2 m^{2} n(16)\right] Y_{1 C S}+m^{2} n(32) R_{C S}\right\} \\
& =\mathrm{Y}_{1 \mathrm{CS}}-\left(\mathrm{Y}_{1 \mathrm{CS}}-\mathrm{R}_{\mathrm{CS}}\right) /(2 \mathrm{n})
\end{aligned}
$$

Esta fórmula (Ecuación 11) es la que se generaría a partir del enfoque de Wright (1981) para 2n progenitores. Lo interesante es que en la derivación aquí realizada se partió de la VS formada por $n$ progenitores (las $n$ cruzas dobles).

Mediante procedimientos similares a los que condujeron a la fórmula de predicción de $\mathrm{Y}_{2 \mathrm{CD}}^{\mathrm{CD}}$ (Ecuación 10), a continuación se deriva una fórmula similar, pero en términos de cruzas simples y líneas. Considérese las expresiones

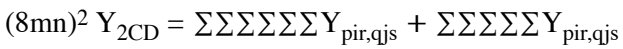

$$
\begin{aligned}
& \text { y }
\end{aligned}
$$

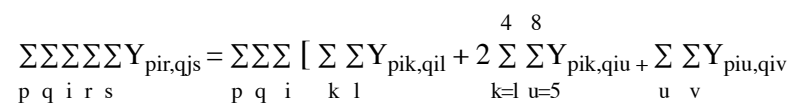

Los valores genotípicos incluidos en los términos

$$
\begin{aligned}
& 48 \\
& \sum \sum \sum \sum \sum \sum \mathrm{Y}_{\text {pir,qjs }} \quad \text { y } \quad \sum \sum \sum \sum \sum \mathrm{Y}_{\text {pik,qiu }} \\
& \text { pq i j j s } \quad \text { p q i k }=1 \mathrm{u}=5
\end{aligned}
$$

pueden ser visualizados como los correspondientes a los genotipos que producen todas las cruzas entre cruzas simples diferentes. Además, de acuerdo con la Ecuación 9, de los $32 \mathrm{~m}^{2} \mathrm{n}$ valores genotípicos que forman los términos

$$
\begin{aligned}
& \sum \sum \sum \sum \sum \mathrm{Y}_{\text {pik,qil }} \text { y } \sum \sum \sum \sum \sum \mathrm{Y}_{\text {pik,qiu }} \text {, } \\
& \text { pqikl pqi u }
\end{aligned}
$$

$16 \mathrm{mn}$ resultan de la autofecundación de los individuos que representan a las líneas. Los restantes $16 \mathrm{mn}(2 \mathrm{~m}-1)$ corresponden a genotipos producidos por 
los cruzamientos entre individuos de una misma cruza simple. Si las medias de estos dos grupos se representan por $\mathrm{S}_{\mathrm{L}} \mathrm{y} \mathrm{R}_{\mathrm{CS}}$, la media genotípica de la VS será expresable como:

$$
\begin{aligned}
& \mathrm{Y}_{2 \mathrm{CD}}^{\mathrm{CS}}=\frac{1}{(8 \mathrm{mn})^{2}}\left[\begin{array}{c}
\sum \sum \sum \sum \sum \sum \mathrm{Y}_{\text {pir,qjs }} \\
{\left[\begin{array}{l}
2 \sum \mathrm{r} \mathrm{s} \\
2 \sum \sum \sum \sum \mathrm{Y}_{\text {pik,qiu }}
\end{array}\right.}
\end{array}\right. \\
& \begin{array}{cc}
+\sum \sum \sum \sum \sum \mathrm{Y}_{\text {pik,qil }} & +\sum \sum \sum \sum \sum \mathrm{Y}_{\text {pik,qiv }} \\
\text { p q i k 1 } & \text { p q i u v }
\end{array} \\
& =\underline{1}\left\{\left[64 m^{2} n(n-1)+32 m^{2} n\right] Y_{1 C S}+16 m n(2 m-1) R_{C S}+16 m n S_{L}\right\} \\
& (8 \mathrm{mn})^{2} \\
& =\mathrm{Y}_{1 \mathrm{CS}}-\left(\mathrm{Y}_{1 \mathrm{CS}}-\mathrm{R}_{\mathrm{CS}}^{\prime}\right) /(2 \mathrm{n})-\left(\mathrm{R}_{\mathrm{CS}}^{\prime}-\mathrm{S}_{\mathrm{L}}\right) /(4 \mathrm{mn})
\end{aligned}
$$

Por otra parte, se consideró que la progenie de la cruza entre dos cruzas dobles diferentes es la misma que la que resulte de las 16 cruzas posibles entre cada una de las cuatro líneas de una cruza doble con las cuatro líneas de la otra. Además, el apareamiento aleatorio en aislamiento entre los individuos de una cruza doble debe generar un arreglo genotípico igual al de la población que producen las 12 cruzas (directas y recíprocas) entre las cuatro líneas de esa cruza doble y el apareamiento aleatorio en aislamiento de los individuos de cada una de estas cuatro líneas. En una VS formada con $n$ cruzas dobles, hay $n(n-1)$ cruzas entre ellas y $n$ poblaciones que forman el apareamiento aleatorio en aislamiento de los individuos de cada cruza doble. En el contexto de estas consideraciones, a continuación se muestra la derivación analítica de la media genotípica de la VS expresada en términos de las 4n líneas. Con base en la Ecuación 9,

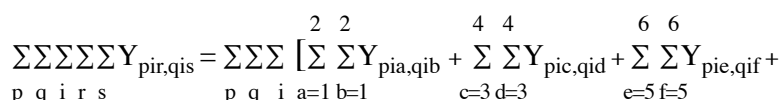

$$
\begin{aligned}
& \text { ( }
\end{aligned}
$$

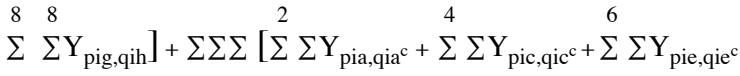

$$
\begin{aligned}
& \mathrm{g}=7 \mathrm{~h}=7 \quad \text { p q i } \quad \mathrm{a}=1 \mathrm{a}^{\mathrm{c}} \quad \mathrm{c}=3 \mathrm{c}^{\mathrm{c}} \quad \mathrm{e}=5 \mathrm{e}^{\mathrm{c}} \\
& 8 \\
& \left.+\sum \sum \mathrm{Y}_{\mathrm{pig}, \mathrm{qig}}\right] \\
& \mathrm{g}=7 \mathrm{gc}
\end{aligned}
$$

Supóngase ahora que $\mathrm{Y}_{1 \mathrm{~L}}$ y $\mathrm{R}_{\mathrm{L}}$ son, respectivamente, la media genotípica de todas las progenies de las cruzas entre dos líneas diferentes y la media de las poblaciones que resultan del apareamiento aleatorio en aislamiento de los individuos de cada línea. Así,

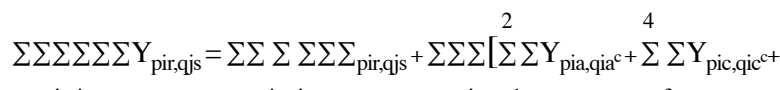

$$
\begin{aligned}
& \text { pqijrs pqizjrs pqia } a=1 a^{c} \quad c=3 c^{c} \\
& \left.+\stackrel{6}{\sum} \sum \mathrm{Y}_{\text {pie,qiec }}+\sum \sum \mathrm{Y}_{\text {pig,qigc }}\right]+ \\
& \mathrm{e}=5 \mathrm{e}^{\mathrm{c}} \quad \mathrm{g}=7 \mathrm{gc}
\end{aligned}
$$

$$
\begin{aligned}
& \begin{array}{llllllll}
22 & 4 & 4 & 6 & 6 & 8 & 8
\end{array} \\
& +\sum \Sigma \Sigma\left[\sum \sum \mathrm{Y}_{\text {pia,qib }}+\sum \sum \mathrm{Y}_{\text {pic,qid }}+\sum \sum \mathrm{Y}_{\text {pic,qif }}+{ }^{8} \sum \mathrm{Y}_{\text {pig,qih }}{ }^{+}\right. \\
& \text {p q i } a=1 b=1 \quad \mathrm{c}=3 \mathrm{~d}=3 \quad \mathrm{e}=5 \mathrm{f}=5 \quad \mathrm{~g}=7 \mathrm{~h}=7 \\
& =\left[(8 m)^{2} n(n-1)+48 m^{2} n\right] Y_{1 L}+16 m^{2} n R_{L}
\end{aligned}
$$

Por lo tanto (Ecuación 6), la media genotípica de la VS $\left(\mathrm{Y}_{2 \mathrm{CD}}^{\mathrm{L}}\right)$, puede expresarse en términos de las $4 \mathrm{n}$ líneas que forman las $\mathrm{n}$ cruzas dobles en la forma

$$
\begin{aligned}
\mathrm{Y}_{2 \mathrm{CD}}^{\mathrm{L}} & =\frac{\left[(8 \mathrm{~m})^{2} \mathrm{n}(\mathrm{n}-1)+48 \mathrm{~m}^{2} \mathrm{n}\right] \mathrm{Y}_{1 \mathrm{~L}}+16 \mathrm{~m}^{2} \mathrm{n} \mathrm{R}_{\mathrm{L}}}{(8 \mathrm{mn})^{2}} \\
& =\mathrm{Y}_{1 \mathrm{~L}}+\left(\mathrm{R}_{\mathrm{L}}-\mathrm{Y}_{1 \mathrm{~L}}\right) /(4 \mathrm{n})
\end{aligned}
$$

La Ecuación 13 es la que se obtendría para una VS de 4n líneas según el método de Wright (1981) y, en el caso de líneas completamente homocigóticas no emparentadas, con el de Wright (1922). Así, sin efectos maternos, la estimación (o predicción) de la media de la VS se puede basar en la evaluación de campo de las $4 \mathrm{n}$ poblaciones que forma el apareamiento aleatorio en aislamiento de los individuos de cada línea y las $2 n(4 n-1)$ cruzas simples directas que se puede hacer con las 4 n líneas.

Con respecto a las $4 n$ líneas, la media genotípica de las poblaciones que forma el apareamiento aleatorio en aislamiento de los individuos de cada línea es

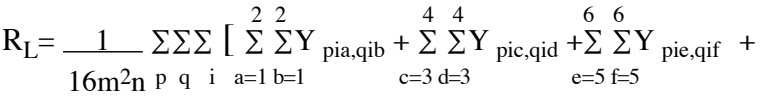

$$
\begin{aligned}
& \left.+\sum_{\mathrm{g}=7 \mathrm{~h}=7}^{8} \sum_{\text {pig,qih }}^{8}\right] \\
& =\frac{1}{16 \mathrm{~m}^{2} \mathrm{n}}\left\{\sum _ { \mathrm { p } \neq \mathrm { q } } \sum \sum _ { \mathrm { i } } \left[{ }^{2} \sum_{\mathrm{a}=1}^{2} \sum_{\mathrm{b}=1}^{2} \mathrm{Y}_{\text {pia,qib }}+\sum_{\mathrm{c}=3}^{4} \sum_{\mathrm{d}=3}^{4} \mathrm{Y}_{\text {pic,qid }}+\sum_{\mathrm{e}=5}^{6} \sum_{\mathrm{f}=5}^{6} \mathrm{Y}_{\text {pie,qif }}+\right.\right. \\
& \left.+\sum_{\mathrm{g}=7}^{8} \sum_{\mathrm{h}=7}^{8} \mathrm{Y}_{\text {pig,qih }}\right]
\end{aligned}
$$

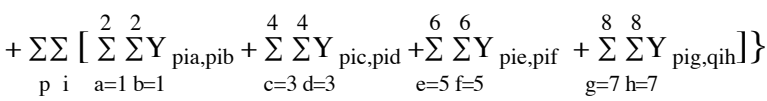

La primera parte de $\mathrm{R}_{\mathrm{L}}$ está constituida por los valores genotípicos de todos los individuos resultantes de todas las cruzas entre los representantes de cada línea; y la segunda parte está formada por los que resultan de la autofecundación de los individuos de cada línea. Así, si y $R_{L}^{\prime}$ y $S_{L}$ representan la media de estos dos grupos de individuos, respectivamente, se obtendrá que

$$
\mathrm{R}_{\mathrm{L}}=\frac{16 \mathrm{~m}(\mathrm{~m}-1) n}{16 \mathrm{~m}^{2} \mathrm{n}} \mathrm{R}_{\mathrm{L}}^{\prime}+\frac{16 \mathrm{mn}}{16 \mathrm{~m}^{2} \mathrm{n}} \mathrm{S}_{\mathrm{L}}=\frac{\mathrm{m}-1}{\mathrm{~m}} \mathrm{R}_{\mathrm{L}}^{\prime}+\frac{1}{\mathrm{n}} \mathrm{S}_{\mathrm{L}}
$$

La substitución de este resultado en la Ecuación 13 produce la ecuación 


$$
\begin{aligned}
& Y_{2 C D}^{L}=\frac{4 n-1}{4 n} Y_{1 L}+\frac{m-1}{4 n m} R_{L}^{\prime}+\frac{1}{4 n m} S_{L} \\
& =Y_{1 L}-\left(Y_{1 L}-R_{L}^{\prime}\right) / 4 n-\left(R_{L}^{\prime}-S_{L}\right) / 4 m n
\end{aligned}
$$

Se han derivado seis fórmulas para la media genotípica de una VS cuyos progenitores son n híbridos de cruza doble. Cada una es base para una fórmula de predicción de la media de la VS. El predictor asociado a cualquiera de estas fórmulas es lo que resulta de substituir en su lado derecho las medias genotípicas por sus respectivas medias fenotípicas, que son las medias experimentales de las familias correspondientes. En virtud de que estas medias fenotípicas están integradas por la media genotípica y un componente aleatorio de error cuyo valor esperado es cero, los seis predictores deben ser estimadores insesgados de la media de la VS.

Por otro lado, VS sujeta a predicción no es sólo la que formaría un conjunto particular de cruzas dobles; sino que también sería formada por cualquier conjunto de $n$ cruzas dobles que se pueda construir con las mismas 4 n líneas, siempre y cuando cada línea participe sólo una vez y sólo en una cruza doble. Asimismo, también sería la que formaran las $4 \mathrm{n}$ líneas, o cualquier conjunto de $2 n$ cruzas simples construidas con esas $4 n$ líneas, de manera que cada línea participe una sola vez en sólo una cruza simple. Esta multiplicidad de conjuntos de progenitores para una misma VS se debe a que, en ausencia de agentes que cambien las frecuencias génicas, los individuos de cualquiera de estos conjuntos de progenitores debe producir el mismo arreglo gamético y, en consecuencia, la misma población o variedad sintética. Sin embargo, desde el punto de vista de requerimientos de recursos sí existen diferencias importantes entre tipos de progenitores de la misma VS.

El trabajo de formación y evaluación de materiales es diferente para cada tipo de progenitores y debe ser un criterio para seleccionar una fórmula de predicción. Por ejemplo, según la Ecuación 8, la predicción de la media genotípica de la VS requiere la disponibilidad y evaluación de campo de las $n(n-1) / 2$ cruzas directas entre cruzas dobles (sin efectos maternos la media genotípica de las cruzas dobles debe ser igual a la de sus recíprocas) $\mathrm{y}$ de las $\mathrm{n}$ poblaciones que forma el apareamiento aleatorio en aislamiento de los $\mathrm{m}$ individuos de cada cruza doble. Con estas evaluaciones se estima, respectivamente, y con tales estimaciones se hace la predicción de acuerdo con la Ecuación 8. Esta predicción requiere evaluar $n(n+1) / 2$ materiales, que son considerablemente menos si se compara con $\operatorname{los} n(2 n+1)$ o $2 n(4 n+1)$ materiales que requiere la predicción basada en las $2 \mathrm{n}$ cruzas simples o en las $4 \mathrm{n}$ líneas según las Ecuaciones 11 y 13, respectivamente. En la realidad, estas diferencias en números de tratamientos no se deben interpretar só- lo en un contexto cuantitativo o gradual. La opción económicamente más factible sería la de la predicción basada en las cruzas dobles (Ecuación 8), por el menor número de materiales que habría que evaluar.

Otro criterio que se debe considerar para seleccionar un método de predicción es la facilidad con que se pueda preparar los materiales a evaluar. Por ejemplo, según la Ecuación 10, la predicción requiere estimar $\mathrm{R}_{\mathrm{CD}}, \mathrm{Y}_{1 \mathrm{CD}} \mathrm{y} \mathrm{S}_{\mathrm{L}}$. Sin embargo, la estimación de $\mathrm{R}_{\mathrm{CD}}$ puede dificultarse puesto que implica que cada una de las $m$ plantas que representan cada cruza doble sea cruzada con cada una de las m-1 restantes, y la disponibilidad de polen (por el lado del macho), de inflorescencias femeninas (por el lado de la hembra) y/o de mano de obra pueden ser insuficientes. Por estas razones, las fórmulas de predicción que involucren la estimación de medias similares $\mathrm{R}_{\mathrm{CD}} \mathrm{y}_{\mathrm{R}}{ }_{\mathrm{L}}$ (Ecuaciones 12 y 14), también podrían tener un valor aplicado restringido. Sin embargo, es conveniente su estudio para determinar su valor según otros criterios y así tener una evaluación más completa de estos métodos.

Otro criterio que contribuye a la caracterización de las fórmulas de predicción es la varianza del predictor. Con base en las cruzas directas entre progenitores, a continuación se mostrará la varianza de cada uno de los predictores derivados. $\mathrm{Si \sigma}^{2}$ es la varianza de error del experimento en que se evalúa los materiales genéticos, la varianza del predictor, construido con base en la Ecuación 8, pero con sólo las cruzas directas y en términos de una sola repetición es (Wricke y Weber 1986, Sahagún-Castellanos 1998).

$$
\operatorname{Var}\left[\hat{\mathrm{Y}}_{2 \mathrm{CD}}\right]=\frac{2 \mathrm{n}-1}{\mathrm{n}^{3}} \sigma^{2}
$$

A su vez, las varianzas de los predictores de la VS basados tanto en la evaluación de las poblaciones que produce el apareamiento aleatorio en aislamiento entre los individuos que representa cada progenitor y los que producen las cruzas directas entre progenitores, sean cruzas simples (Ecuación 11) o líneas, son respectivamente:

$$
\begin{aligned}
\operatorname{Var}[\hat{\mathrm{Y} C S}] & =\left[\frac{\left(\frac{2 \mathrm{n}-1)^{2}}{(2 \mathrm{n})^{2}} \frac{2}{2 \mathrm{CD}(2 \mathrm{n}-1)}+\frac{1}{(2 n)^{3}}\right] \sigma^{2}=\frac{(2 \mathrm{n}-1)+1}{(2 \mathrm{n})^{3}} \sigma^{2}}{}\right. \\
& =\frac{4 \mathrm{n}-1}{8 \mathrm{n}^{3}} \sigma^{2} \\
\mathrm{y} &
\end{aligned}
$$

$$
\begin{aligned}
\operatorname{Var}\left[\hat{Y^{L}}\right] & =\left[\frac{(4 n-1)^{2}}{(4 n)^{2}} \frac{2}{4 n(4 n-1)}+\frac{1}{(4 n)^{3}}\right] \sigma^{2}=\left[2\left(\frac{4 n-1)+1}{(4 n)^{3}}\right] \sigma^{2}\right. \\
& =\frac{8 n-1}{64 n^{3}} \sigma^{2}
\end{aligned}
$$


Respecto a los predictores correspondientes a las Ecuaciones 10, 12 y 14, sus varianzas son respectivamente:

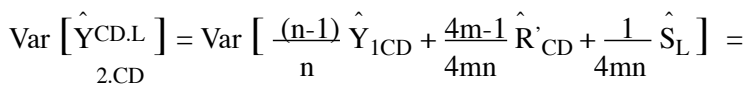

$$
\begin{aligned}
& {\left[\frac{2 n-1}{n^{3}}-\frac{m-5 / 32}{2 m^{2} n^{3}}\right] \sigma^{2}} \\
& \operatorname{Var}\left[\hat{\mathrm{Y}}_{2 . \mathrm{CD} . \mathrm{L}}\right]=\operatorname{Var}\left[\frac{2 \mathrm{n}-1}{2 \mathrm{n}} \hat{\mathrm{Y}}_{1 \mathrm{CS}}+\frac{2 \mathrm{~m}-1}{4 \mathrm{mn}} \hat{\mathrm{R}}_{\mathrm{CD}}^{\prime}+\frac{1}{4 m n} \hat{\mathrm{S}}_{\mathrm{L}}\right]= \\
& {\left[\frac{4 n-1}{(2 n)^{3}}-\frac{m-0,375}{m^{2}(2 n)^{3}}\right] \sigma^{2}} \\
& \operatorname{Var}\left[\hat{\mathrm{Y}}_{2 . \mathrm{CD}}^{\mathrm{L} . \mathrm{L}}\right]=\operatorname{Var}\left[\frac{4 \mathrm{n}-1}{4 \mathrm{n}} \hat{\mathrm{Y}}_{1 \mathrm{~L}}+\frac{\mathrm{m}-1}{4 m n} \hat{\mathrm{R}}_{\mathrm{CD}}^{\prime}+\frac{1}{4 m n} \hat{\mathrm{S}}_{\mathrm{L}}\right]= \\
& {\left[\frac{8 n-1}{(4 n)^{3}}-\frac{m-1}{(2 m)^{2}(2 n)^{3}}\right] \sigma^{2}}
\end{aligned}
$$

De las varianzas descritas en las Ecuaciones 15, 16 y 17, la de la predicción con base en las 4n líneas (Ecuación 17) es la más pequeña y la mayor es la de cruzas dobles (Ecuación 15). Sin embargo, en requerimientos de unidades experimentales la evaluación de cruzas y sus líneas es la más demandante. Una comparación más conveniente, en términos de administración de recursos, es la que se hizo en la base de un mismo número de unidades experimentales (Sahagún-Castellanos, 1998). Una forma de lograr esta comparación se obtiene cuando en los casos en que los progenitores son cruzas dobles y cruzas simples se tuvieran los incrementos en repeticiones necesarios para que en los tres casos la evaluación se hiciera en las $4 n(4 n+1) / 2$ unidades experimentales que requiere la evaluación de los materiales asociados al predictor de la VS cuyos progenitores son las líneas (Ecuación 17). Las varianzas de estos nuevos predictores, para cruzas dobles y cruzas simples fueron, respectivamente:

$$
\begin{aligned}
& \operatorname{Var}\left[\hat{\hat{Y}}_{2 . C D}\right]=\frac{\left[(2 n-1) / n^{3}\right]}{[4(4 n+1)] /(n+1)} \underline{\sigma}_{-}^{2}=\frac{(2 n-1)(n+1)}{4 n^{3}(4 n+1)} \sigma^{2} \\
& y \\
& \left.\operatorname{Var}\left[\hat{\hat{Y}}_{2 . C D}^{c s}\right]=\frac{\left[(4 n-1) /\left(8 n^{3}\right)\right.}{[2(4 n+1)] /(2 n+1)}\right]_{-}{ }^{2}=\frac{(4 n-1)(2 n+1)}{2[4 n+1] 8 n^{3}} \sigma^{2}
\end{aligned}
$$

El Cuadro 1 muestra las varianzas de los estimadores basados en la evaluación en una repetición de las progenies producidas por las cruzas directas entre los progenitores y por el apareamiento aleatorio en aislamiento de los individuos de cada progenitor para varios números de progenitores (Ecuaciones 15, 16 y 17). Para cada valor de $\mathrm{n}$ las varianzas menor y mayor ocurrieron cuando los progenitores fueron las líneas (L) y las cruzas dobles (CD), respectivamente (Ecuaciones $17 \mathrm{y}$ 15). Sin embargo, si bien con CD el estimador tiene la mayor varianza (menor precisión en la estimación), éste requiere el menor número de parcelas para la evaluación de materiales.

Respecto a la evaluación en una repetición, en la evaluación de materiales en un mismo número de parcelas (las requeridas por L), las relaciones entre las magnitudes de las varianzas de los estimadores, en general, se mantuvieron, aunque las diferencias se redujeron, particularmente para valores grandes de $n$ (Cuadro 1). Las diferencias que sí son de consideración, sin em-

\begin{tabular}{|c|c|c|c|c|c|c|c|c|c|}
\hline \multirow[t]{2}{*}{$\mathbf{n}$} & \multicolumn{3}{|c|}{ Número de materiales } & \multicolumn{3}{|c|}{$\begin{array}{c}\text { Coeficientes de la varianza } \\
\text { del estimador } \mathbf{U R}^{\ddagger}\end{array}$} & \multicolumn{3}{|c|}{$\begin{array}{l}\text { Coeficientes de varianza } \\
\text { del estimador INPI }\end{array}$} \\
\hline & CD & CS & $\mathbf{L}$ & CD & CS & $\mathbf{L}$ & CD & CS & $\mathbf{L}$ \\
\hline 1 & 1 & 3 & 10 & 1.0000 & 3.750 & 1.094 & 1.000 & 1.125 & 1.094 \\
\hline 2 & 3 & 10 & 36 & 3.750 & 1.094 & 293 & 313 & 304 & 293 \\
\hline 3 & 6 & 21 & 78 & 1.852 & 509 & 133 & 143 & 137 & 133 \\
\hline 4 & 10 & 36 & 136 & 1.094 & 293 & 76 & 80 & 78 & 76 \\
\hline 5 & 15 & 55 & 210 & 720 & 190 & 48 & 51 & 50 & 48 \\
\hline 6 & 21 & 78 & 300 & 509 & 133 & 32 & 36 & 35 & 32 \\
\hline 8 & 36 & 136 & 528 & 293 & 76 & 19 & 20 & 20 & 19 \\
\hline 10 & 55 & 210 & 820 & 190 & 49 & 12 & 13 & 12 & 12 \\
\hline
\end{tabular}
bargo, son las que se observan entre las varianzas de los dos tipos de evaluación (una repetición e igualdad de parcelas), excepto cuando las líneas (L) fueron los progenitores de la VS. Estas diferencias se relacionan

Cuadro 1. Número de materiales evaluados y coeficientes $\left(\mathrm{X} 10^{4}\right)$ de la varianza del predictor de la media genotípica de una variedad sintética construida con $\mathrm{n}$ cruzas dobles (CD), $2 \mathrm{n}$ cruzas simples (CS) ó 4n líneas (L) [Ecuaciones 15, 16 y 17].

$\ddagger$ UR: La evaluación se hizo en una repetición.

IJ INP: La evaluación se hizo en igual número de parcelas, 2n(4n+1). 
básicamente con las diferencias en los números de parcelas utilizadas para la evaluación; en la medida en que más se incrementó el número de parcelas mayor fue la disminución de la varianza.

Las ecuaciones 18,19 y 20 muestran las varianzas de los predictores basados en CD, CS y L, respectivamente, que requieren la evaluación de las progenies que producen las autofecundaciones de las líneas y las cruzas entre progenitores de cada tipo y entre los individuos de cada progenitor. Las magnitudes de estas varianzas para varios valores de $\mathrm{m}$ (número de individuos por progenitor) y n siempre fueron menores que las correspondientes a los predictores de las Ecuaciones 15, 16 y 17, basados en la evaluación de progenies generadas por las cruzas directas entre progenitores y por el apareamiento aleatorio en aislamiento de los individuos de cada progenitor (Cuadros 1, 2 y 3). Las reducciones mayores y menores ocurrieron cuando los progenitores fueron cruzas dobles y líneas, respectivamente. Sin embargo, la reducción de varianzas por esta vía puede resultar poco atractiva por las dificultades que pueden presentarse en la construcción de las poblaciones asociadas a $\mathrm{R}_{\mathrm{CD}}, \mathrm{R}_{\mathrm{Cs}} \mathrm{y} \mathrm{R}_{\mathrm{L}}$. Respecto a este particular, se ha considerado que la media de estas poblaciones es la media de los progenitores (Márquez 1992b). Sin embargo, el predictor que se construyó con base en esta consideración tiene un sesgo de $(\mathrm{m}-1)\left(\mathrm{Y}_{0}-\mathrm{R}^{\prime}\right) / \mathrm{mn}$ en donde R' es la media de las poblaciones que forman las cruzas inter se de los individuos de cada progenitor (Sahagún 1998). Este sesgo se reduce a medida que mn es mayor o que $\mathrm{Y}_{0}$ - R' es más pequeña; y es cero cuando, por ejemplo, los progenitores son líneas homocigóticas caso en el que no tendría sentido la estimación en que se incluya $R_{L}^{\prime}$ y $S_{L}$ ya que ambos serían iguales.
Las varianzas de los predictores correspondientes a las Ecuaciones 10, 12 y 14 también fueron expresadas en términos de un mismo número de parcelas (mediante el incremento necesario en número de repeticiones que demande el uso de $n(4 n+3) / 2$ parcelas, que son las que requirió para una repetición la evaluación de materiales correspondientes al estimador $\hat{Y}_{C D}^{\text {L.L }}$ (Ecuación 20). Estas varianzas (Cuadro 3) también disminuyeron, a excepción de las de $\hat{Y}_{\mathrm{CD}}^{\mathrm{LL}}$, respecto a las correspondientes del Cuadro 2, y las menores fueron las correspondientes a $\hat{Y}_{C D}^{L . L}$ en donde, a diferencia de $\hat{Y}_{2 C D}^{C D . L} \quad y$ $\hat{Y}_{2 \mathrm{CD}}^{\text {CS.L }}$ (Ecuaciones 18 y 19), las varianzas, en general, no crecieron a medida que $\mathrm{m}$ fue mayor. $\mathrm{Y}$ las diferencias entre las varianzas para los diferentes valores de $\mathrm{m}$ se redujeron a medida que $n$ fue mayor; desde $n=6$ en adelante las varianzas para los cuatro valores de $\mathrm{m}$ fueron iguales. Con base en las varianzas solamente, el mejor de los tres estimadores (Ecuaciones 10, 12 y 14) es $\hat{\mathrm{Y}}_{2 \mathrm{CD}}^{\mathrm{LL}}$; sus varianzas son aproximadamente de una tercera a una quinta parte de las de $\hat{\mathrm{Y}}_{2 \mathrm{CD}}^{\mathrm{CD} . \mathrm{y}}$ y de poco menos que la mitad de la de $\hat{Y}_{2 C D}^{\text {CS.L }}$. Esto significa que, por ejemplo, si para que $\hat{Y}_{2 C D}^{C D L}$ tenga la misma precisión se requiere una evaluación en un número de repeticiones que sea de tres a cinco veces, según corresponda, mayor que las que se use para el caso de $\hat{\mathrm{Y}}_{2 \mathrm{CD}}^{\mathrm{CS} \text {. }}$.

Las varianzas del Cuadro 3; sin embargo no son apropiadamente comparables con las correspondientes del Cuadro 1 (las del estimador INP) pues están calculadas con base en diferentes números de parcelas. Las varianzas de los estimadores $\hat{\mathrm{Y}}_{2 \mathrm{CD}}^{\mathrm{CD}}, \hat{\mathrm{Y}}_{2 \mathrm{CD}}^{\mathrm{CS} \text { y }} \hat{\mathrm{Y}}_{\text {2CD }}^{\mathrm{LCL}}$ (Cuadro 3) que sí son comparables con las de los estimadores $\hat{\mathrm{Y}}_{2 \mathrm{CD}}, \hat{\mathrm{Y}}_{2 \mathrm{CD}}^{\mathrm{CS}} \mathrm{y} \hat{\mathrm{Y}}_{2 \mathrm{CD}}^{\mathrm{L}}$ (Cuadro 1) para un mismo número de parcelas son las del Cuadro 4. Los valores relativos de las varianzas correspondientes de estos dos cuadros implican que los estimadores $\hat{\mathrm{Y}}_{2 \mathrm{CD}}^{\mathrm{CD}}, \hat{\mathrm{Y}}_{2 \mathrm{CD}}^{\mathrm{CS}}$ y $\hat{\mathrm{Y}}_{2 \mathrm{CDS}}^{\mathrm{LL}} \mathrm{L}$ más

Cuadro 2. Coeficiente $\left(\mathrm{X} 10^{4}\right)$ de la varianza del predictor ${ }^{\Im}$ de una variedad sintética construida con $\mathrm{n}$ cruzas dobles (CD), 2n cruzas simples (CS) o 4n líneas (L) con 1, 2, 4 ó 6 plantas por progenitor (Ecuaciones 18, 19 y 20). La evaluación se hizo en una repetición.

\begin{tabular}{|c|c|c|c|c|c|c|c|c|c|c|c|c|}
\hline \multirow[b]{2}{*}{ n } & \multicolumn{4}{|c|}{ CD } & \multicolumn{4}{|c|}{ CS } & \multicolumn{4}{|c|}{$\mathbf{L}$} \\
\hline & 1 & 2 & 4 & 6 & 1 & 2 & 4 & 6 & 1 & 2 & 4 & 6 \\
\hline 1 & 5.781 & 7.695 & 8.799 & 9.188 & 2.969 & 3.242 & 3.467 & 3.555 & 1.094 & 1.016 & 1.035 & 1.050 \\
\hline 2 & 3.223 & 3.642 & 3.600 & 3.649 & 996 & 1.030 & 1.058 & 1.069 & 293 & 283 & 286 & 288 \\
\hline 3 & 1.696 & 1.766 & 1.807 & 1.822 & 480 & 490 & 499 & 502 & 133 & 130 & 131 & 131 \\
\hline 4 & 1.028 & 1.058 & 1.075 & 1.081 & 281 & 285 & 289 & 290 & 76 & 74 & 75 & 75 \\
\hline 5 & 686 & 702 & 710 & 714 & 184 & 186 & 188 & 188 & 49 & 48 & 48 & 48 \\
\hline 6 & 490 & 499 & 504 & 506 & 129 & 131 & 132 & 132 & 34 & 34 & 34 & 34 \\
\hline 8 & 285 & 288 & 291 & 291 & 74 & 75 & 75 & 75 & 19 & 19 & 19 & 19 \\
\hline 10 & 186 & 188 & 189 & 189 & 48 & 48 & 48 & 49 & 12 & 12 & 12 & 12 \\
\hline
\end{tabular}

I El predictor se construyó con base en las cruzas directas entre progenitores y entre los m individuos de cada progenitor y en las poblaciones que forma el apareamiento aleatorio en aislamiento entre los $\mathrm{m}$ representantes de cada progenitor. 
Cuadro 3. Coeficientes $\left(\mathrm{X} 10^{4}\right)$ de la varianza del predictor de la media genotípica de una VS construida con $\mathrm{n}$ cruzas dobles (CD), 2n cruzas simples (CS) o 4n líneas (L) con 1, 2, 4, 6 plantas por progenitor (Ecuaciones $18,19,20)$. En todos los casos la evaluación se hizo en $2 n(4 n+3)$ parcelas.

\begin{tabular}{|c|c|c|c|c|c|c|c|c|c|c|c|c|}
\hline \multirow[b]{2}{*}{ n } & \multicolumn{4}{|c|}{ CD } & \multicolumn{4}{|c|}{ CS } & \multicolumn{4}{|c|}{$\mathbf{L}$} \\
\hline & 1 & 2 & 4 & 6 & 1 & 2 & 4 & 6 & 1 & 2 & 4 & 6 \\
\hline 1 & 3.303 & 4.397 & 5.028 & 5.250 & 2.121 & 2.316 & 2.476 & 2.539 & 1.094 & 1.016 & 1.035 & 1.050 \\
\hline 2 & 1.465 & 1.655 & 1.636 & 1.659 & 634 & 655 & 673 & 680 & 293 & 283 & 286 & 288 \\
\hline 3 & 678 & 706 & 723 & 729 & 288 & 294 & 299 & 301 & 133 & 130 & 131 & 131 \\
\hline 4 & 379 & 390 & 396 & 398 & 163 & 165 & 167 & 168 & 76 & 74 & 75 & 75 \\
\hline 5 & 239 & 244 & 247 & 248 & 104 & 105 & 106 & 106 & 49 & 48 & 48 & 48 \\
\hline 6 & 163 & 166 & 168 & 169 & 72 & 73 & 73 & 73 & 34 & 34 & 34 & 34 \\
\hline 8 & 81 & 82 & 83 & 83 & 40 & 41 & 41 & 41 & 19 & 19 & 19 & 19 \\
\hline 10 & 48 & 48 & 48 & 48 & 26 & 26 & 26 & 26 & 12 & 12 & 12 & 12 \\
\hline
\end{tabular}

Cuadro 4. Coeficiente de la varianza $\left(\mathrm{X} 10^{4}\right)$ de los estimadores (FPR) $\hat{\mathrm{Y}}_{2 \mathrm{CD}}^{\mathrm{CD}}, \hat{\mathrm{Y}}_{2 \mathrm{CD}}^{\mathrm{CS} . \mathrm{L}}$ y $\hat{\mathrm{Y}}_{2 \mathrm{CD}}^{\mathrm{LL}}($ Ecuaciones 18,19 y 20$)$ para el mismo número de parcelas que el utilizado en el caso de los estimadores (PF) $\hat{\mathrm{Y}}_{2 \mathrm{CD}}, \hat{\mathrm{Y}}_{2 \mathrm{CD}}^{\mathrm{CS}}$ y $\hat{\mathrm{Y}}_{2 \mathrm{CD}}^{\mathrm{L}}$ eficiencias relativas entre los estimadores FPR y los PF.

\begin{tabular}{|c|c|c|c|c|c|c|}
\hline \multirow[b]{2}{*}{ n } & \multicolumn{3}{|c|}{ Coeficientes* } & \multicolumn{3}{|c|}{$\begin{array}{c}\text { Eficiencias relativas } \\
\text { (FPR/PF) }\end{array}$} \\
\hline & CD & CS & $\mathbf{L}$ & CD & CS & $\mathbf{L}$ \\
\hline 1 & 714 & 804 & 781 & 1.400 & 1.399 & 1.400 \\
\hline 2 & 256 & 249 & 240 & 1.222 & 1.221 & 1.221 \\
\hline 3 & 124 & 119 & 115 & 1.153 & 1.151 & 1.156 \\
\hline 4 & 72 & 70 & 68 & 1.100 & 1.114 & 1.100 \\
\hline 5 & 47 & 46 & 44 & 1.085 & 1.087 & 1.091 \\
\hline 6 & 33 & 32 & 30 & 1.091 & 1.094 & 1.067 \\
\hline 8 & 19 & 19 & 18 & 1.053 & 1.053 & 1.056 \\
\hline 10 & 12 & 11 & 11 & 1.080 & 1.091 & 1.091 \\
\hline
\end{tabular}

${ }^{*}$ Los coeficientes se obtuvieron dividiendo los del Cuadro 1 (estimador INP) entre $(4 n+3) /(4 n+1)$, en donde $n$ es el número de cruzas dobles.

eficientes que los estimadores $\hat{\mathrm{Y}}_{2 \mathrm{CD}}, \hat{\mathrm{Y}}_{2 \mathrm{CD}}^{\mathrm{CS}}$ y $\hat{\mathrm{Y}}_{2 \mathrm{CD}}^{\mathrm{L}}$ (Ecuaciones 8,11 y 13). La superioridad en eficiencia relativa es de un 8 a un $40 \%$; es decir, para que tengan la misma precisión los dos tipos, los estimadores PF deben tener un número adicional de repeticiones de 8 a 40 $\%$, según el caso.

\section{CONCLUSIONES}

Para las VS medias genotípicas de las variedades sintéticas (VS) formadas con $\mathrm{n}$ cruzas dobles se derivaron seis fórmulas insesgadas de predicción (FP) basadas en la evaluación de materiales derivados a partir de no sólo de las n cruzas dobles (CD), sino también a partir de sus $2 \mathrm{n}$ cruzas simples (CS), y aún de sus $4 \mathrm{n}$ líneas (L). El predictor de la media genotípica de la VS que requiere menos parcelas fue basado en $\mathrm{CD}$, aunque la varianza de este predictor fue la mayor para $\mathrm{n} \geq 2$. Para $\mathrm{n}=1$ y un mismo número de parcelas para cada progenitor, la estimación más precisa fue la basada en CD. Tres FP se basaron en la evaluación de las cruzas directas entre progenitores ( $\mathrm{CD}, \mathrm{CS}$ o $\mathrm{L})$ y de las poblaciones (PAA) que produce el apareamiento aleatorio en aislamiento de los individuos de cada progenitor $(\mathrm{CD}$, CS o L). En las tres FP restantes (FPR) cada PAA se descompuso en dos partes: la formada por autofecundaciones y la restante. Según las varianzas de los estimadores, basándose en un mismo número de unidades experimentales, las FPR tuvieron una eficiencia relativa superior de 8 a $40 \%$ en relación a las FP restantes. Respecto al tipo de progenitores, las varianzas más pequeñas para $\mathrm{n}=2,3,4,5,6,8$ y 10 correspondieron a $\mathrm{L}$.

\section{LITERATURA CITADA}

ALLARD, R. W.; BRADSHAW, A. D. 1964. Implications of genotype-environmental interaction in applied plant breeding. Crop Science 4:503-507.

BUSBICE, T. H. 1969. Inbreeding in synthetic varieties. Crop Science 9:601-604.

BUSBICE, T. H. 1970. Predicting yield of synthetic varieties. Crop Science 10:265-269.

GONZÁLEZ, C.;RON, J.; RAMÍREZ, J. L. 1990. Potencial de rendimiento de cinco híbridos de maíz. Sus cruzas y generaciones avanzadas en el centro de Jalisco. In: Memoria XIII Congreso Nacional de Fitogenética. México. p. 346.

MÁRQUEZ, F. 1992a. Inbreeding and yield prediction in synthetic maize cultivars made with parental lines: I. Basic Methods. Crop Science 32:271-274.

MÁRQUEZ, F. 1992b. On the yield prediction of composite varieties of maize. Maydica 37:271-274. 
SAHAGÚN, J. 1998. Efficiency of three methods for prediction of performance of synthetic varieties. J. Genet. \& Plant Breeding 52:143-149.

SAHAGÚN, J.; VILLANUEVA, C. 1997. Teoría de las variedades sintéticas formadas con híbridos de cruzas simples. Rev. Fitotec. Méx. 20:69-79.

VILLANUEVA, C.; CASTILlO, F.; MOLINA, J. D. 1994. Aprovechamiento de cruzamientos dialélicos entre híbridos comerciales de maíz: Análisis de progenitores y cruzas. Rev. Fitotec. Méx. 17:175-185.
WRICKE, G; WEBER, W. E. 1986. Quantitative genetics and selection in plant breeding. W. G. de Berlin. 406 p.

WRIGHT, S. 1922. The effects of inbreeding and cross-breeding on guinea pigs. Tech. Bull. U.S. Dep. Agric. 1112. Washington D.C.

WRIGHT, A. J. 1981. The quantitative genetics of diploid synthetic varieties. In: Proceedings of the Fourth Meeting of the Section Biometrics in Plant Breeding. A. Gallais (ed). Poitiers, France. 